Supplement of

\title{
A new laser-based and ultra-portable gas sensor for indoor and outdoor formaldehyde (HCHO) monitoring
}

J. D. Shutter et al.

Correspondence to: J. D. Shutter (shutter@g.harvard.edu) 
Table S1. Frequencies and line intensities of HITRAN lines used by HAPP fits

\begin{tabular}{|c|c|c|}
\hline $\begin{array}{l}\text { Wavenumber / } \\
\mathrm{cm}^{-1}\end{array}$ & $\begin{array}{c}\text { Molecular } \\
\text { species }\end{array}$ & $\begin{array}{c}\text { Spectral line intensity }(296 \mathrm{~K}) / \\
\mathrm{cm}^{-1} / \text { molecule } \cdot \mathrm{cm}^{-2}\end{array}$ \\
\hline 2831.2598 & $\mathrm{CH}_{4}$ & $2.863 \mathrm{e}-22$ \\
\hline 2831.2701 & $\mathrm{CH}_{4}$ & $2.308 \mathrm{e}-25$ \\
\hline 2831.2737 & $\mathrm{HCHO}$ & $2.101 \mathrm{e}-20$ \\
\hline 2831.2780 & $\mathrm{CH}_{4}$ & $2.471 \mathrm{e}-22$ \\
\hline 2831.2802 & $\mathrm{CH}_{4}$ & $3.408 \mathrm{e}-25$ \\
\hline 2831.3160 & $\mathrm{CH}_{4}$ & $3.394 \mathrm{e}-23$ \\
\hline 2831.3259 & $\mathrm{HCHO}$ & $2.900 \mathrm{e}-21$ \\
\hline 2831.3366 & $\mathrm{CH}_{4}$ & $2.603 \mathrm{e}-25$ \\
\hline 2831.3501 & $\mathrm{CH}_{4}$ & $3.240 \mathrm{e}-25$ \\
\hline 2831.3550 & $\mathrm{HCHO}$ & $1.420 \mathrm{e}-20$ \\
\hline 2831.3657 & $\mathrm{CH}_{4}$ & $3.587 \mathrm{e}-25$ \\
\hline 2831.4055 & $\mathrm{HDO}$ & $1.812 \mathrm{e}-28$ \\
\hline 2831.4097 & HDO & $1.812 \mathrm{e}-28$ \\
\hline 2831.4209 & $\mathrm{CH}_{4}$ & $1.553 \mathrm{e}-23$ \\
\hline 2831.5393 & НСНО & $5.622 \mathrm{e}-21$ \\
\hline 2831.5534 & $\mathrm{CH}_{4}$ & $8.870 \mathrm{e}-25$ \\
\hline 2831.5576 & HCHO & $5.543 \mathrm{e}-21$ \\
\hline 2831.5616 & $\mathrm{CH}_{4}$ & $1.549 \mathrm{e}-23$ \\
\hline 2831.5801 & $\mathrm{HDO}$ & $4.140 \mathrm{e}-28$ \\
\hline 2831.6413 & HCHO & $5.839 \mathrm{e}-20$ \\
\hline 2831.6879 & $\mathrm{HCHO}$ & $1.499 \mathrm{e}-21$ \\
\hline 2831.6892 & $\mathrm{HCHO}$ & $1.499 \mathrm{e}-21$ \\
\hline 2831.6961 & $\mathrm{CH}_{4}$ & $6.671 \mathrm{e}-25$ \\
\hline 2831.6989 & НСНО & $1.410 \mathrm{e}-20$ \\
\hline 2831.8134 & HCHO & $6.509 \mathrm{e}-21$ \\
\hline 2831.8214 & $\mathrm{CH}_{4}$ & $3.671 \mathrm{e}-24$ \\
\hline 2831.8413 & HDO & $3.014 \mathrm{e}-24$ \\
\hline 2831.8516 & $\mathrm{CH}_{4}$ & $1.983 \mathrm{e}-24$ \\
\hline 2831.8906 & $\mathrm{HDO}$ & $9.812 \mathrm{e}-28$ \\
\hline 2831.8948 & $\mathrm{H}_{2} \mathrm{O}$ & $1.595 \mathrm{e}-28$ \\
\hline 2831.9199 & $\mathrm{CH}_{4}$ & $1.622 \mathrm{e}-21$ \\
\hline 2831.9569 & HDO & $2.713 \mathrm{e}-27$ \\
\hline
\end{tabular}

Spectral lines from the same molecular species are fitted together rather than independently. Lines bolded in blue were used in experiments that utilized ultra-zero air, and it was discovered that all the lines listed produced a more superior fit than only the lines bolded in blue when sampling ambient air. Spectral frequencies and line intensities were accessed using HITRAN on the Web (http://hitran.iao.ru/) (Rothman et al., 2013). 
Table S2. 1 $\sigma$ standard deviation for Aeris sensor at various integration times. All values are in pptv and were obtained by either (1) bubbling ultrazero air $(1000 \mathrm{sccm})$ though water (HDO mode) or (2) adding chemically-pure $99.5 \% \mathrm{CH}_{4}(<1 \mathrm{sccm})$ to $5000 \mathrm{sccm}$ ultra-zero air $\left(\mathrm{CH}_{4}\right.$ mode).

\begin{tabular}{|c|c|c|c|c|}
\hline Integration time $/ \mathrm{s}$ & $\begin{array}{c}\text { ART fit } \\
\text { HDO mode / } \\
\text { pptv HCHO }\end{array}$ & $\begin{array}{c}\text { HAPP fit } \\
\text { HDO mode / } \\
\text { pptv HCHO }\end{array}$ & $\begin{array}{c}\text { Average of the two } \\
\text { HDO modes / } \\
\text { pptv HCHO }\end{array}$ & $\begin{array}{c}\text { HAPP fit } \\
\mathrm{CH}_{4} \text { mode / } \\
\text { pptv } \mathrm{HCHO}\end{array}$ \\
\hline 30 & 1200 & 1000 & 700 & 1350 \\
\hline 60 & 1000 & 800 & 660 & 1100 \\
\hline 120 & 730 & 600 & 530 & 720 \\
\hline 300 & 430 & 380 & 330 & 460 \\
\hline 900 & 230 & 190 & 180 & 320 \\
\hline 1800 & 160 & 140 & 120 & 280 \\
\hline 3600 & 140 & 100 & 100 & 230 \\
\hline 7200 & 160 & 110 & 110 & 140 \\
\hline 20000 & 170 & 160 & 160 & 200 \\
\hline
\end{tabular}

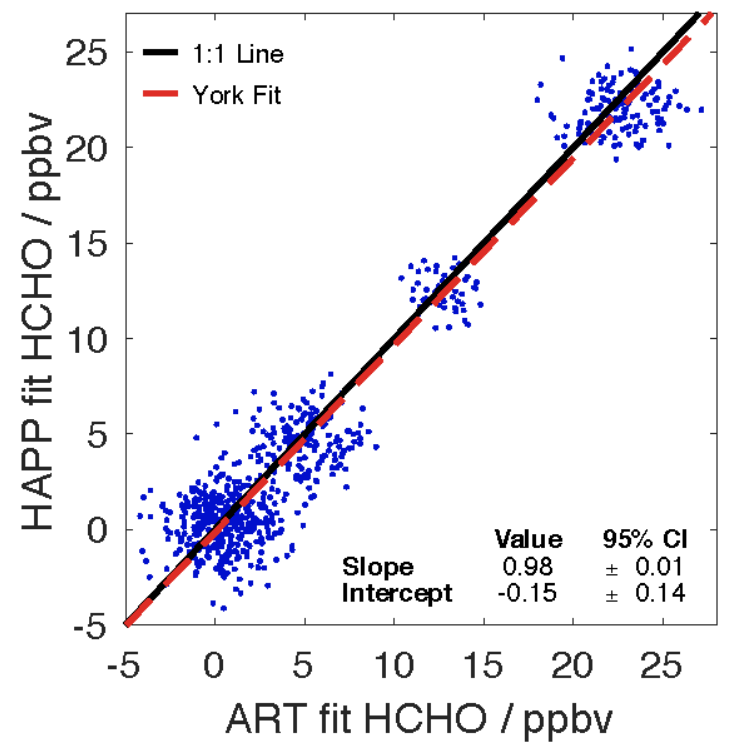

Figure S1. Correlation plot between the Aeris Real-time (ART) fit and the Harvard Aeris-Post Processing $\left(\right.$ HAPP) fit $\left(\mathrm{R}^{2}=0.941\right)$. 


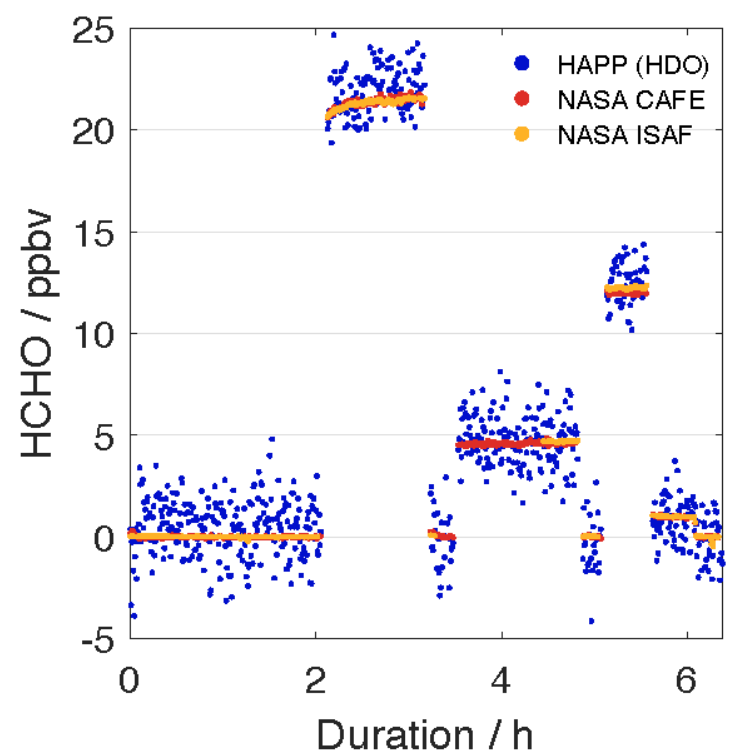

Figure S2. Time series showing the HCHO mixing ratios from the Aeris sensor (HAPP HDO fit) and NASA ISAF and CAFE during a multi-hour stepped intercomparison performed at NASA Goddard in November 2017. All data is reported with an integration time of $30 \mathrm{~s}$.

Rothman, L. S., Gordon, I. E., Babikov, Y., Barbe, A., Benner, D. C., Bernath, P. F., Birk, M., Bizzocchi, L., Boudon, V., Brown, L. R., Campargue, A., Chance, K., Cohen, E. A., Coudert, L. H., Devi, V. M., Drouin, B. J., Fayt, A., Flaud, J.-M., Gamache, R. R., Harrison, J. J., Hartmann, J.-M., Hill, C., Hodges, J. T., Jacquemart, D., Jolly, A., Lamouroux, J., Le Roy, R. J., Li, G., Long, D. A., Lyulin, O. M., Mackie, C. J., Massie, S. T., Mikhailenko, S., Müller, H. S. P., Naumenko, O. V., Nikitin, A. V., Orphal, J., Perevalov, V., Perrin, A., Polovtseva, E. R., Richard, C., Smith, M. 10 A. H., Starikova, E., Sung, K., Tashkun, S., Tennyson, J., Toon, G. C., Tyuterev, V. G. and Wagner, G.: The HITRAN2012 molecular spectroscopic database, J. Quant. Spectrosc. Radiat. Transf., 130, 4-50, doi:10.1016/J.JQSRT.2013.07.002, 2013. 